\title{
Transmissividade Atmosférica da Radiação Global na Região Amazônica de Mato Grosso
}

\author{
Adilson Pacheco de Souza ${ }^{1}$, Tamara Zamadei², Emanoeli Borges Monteiro', \\ Bruno Henrique Casavecchia ${ }^{3}$ \\ ${ }^{1}$ Instituto de Ciências Agrárias e Ambientais, Universidade Federal de Mato Grosso, \\ Sinop, Mato Grosso, Brasil. \\ ${ }^{2}$ Programa de Pós-Graduação em Física Ambiental, Universidade Federal de Mato Grosso, \\ Cuiabá, Mato Grosso, Brasil. \\ ${ }^{3}$ Programa de Pós-Graduação em Ciências Ambientais, Universidade Federal de Mato Grosso, \\ Sinop, Mato Grosso, Brasil.
}

Recebido: 30/11/2015 - Aceito: 21/3/2016

\begin{abstract}
Resumo
Objetivou-se avaliar as variações das médias mensais da radiação global e as frequências do coeficiente de transmissividade atmosférica global (Kt) para 16 municípios da região amazônica do Estado de Mato Grosso. Os dados utilizados foram obtidos pelo Instituto Nacional de Meteorologia, nos períodos de 2008 a 2014. O Kt foi obtido pela razão entre a radiação global e a radiação incidente no topo da atmosfera. Adotou-se a classificação de cobertura de céu em função de Kt para a partição diária em quatro classes: I - céu nublado (Kt $\leq 0,35)$, II - céu parcialmente nublado $(0,35 \leq \mathrm{Kt} \geq 0,55)$, III - céu parcialmente aberto $(0,55<\mathrm{Kt} \geq 0,65)$ e IV - céu aberto $(\mathrm{Kt}>0,65)$. Entre abril e setembro (estação seca) ocorrem maiores índices de transmissividade atmosférica em função da baixa atenuação da radiação solar pela interação com os constituintes atmosféricos, com maiores frequências de condição de céu IV e II. As condições de cobertura de céu são dependentes do comportamento pluviométrico regional e apresentam percentuais médios diferenciados entre municípios do bioma amazônico.
\end{abstract}

Palavras-chave: absorção atmosférica, condições climáticas, índice de claridade, nebulosidade.

\section{Atmospheric Transmissivity of the Global Radiation in the Amazonic Region of Mato Grosso}

\begin{abstract}
It was aimed evaluate the variations of monthly average of global radiation and frequencies of global atmospheric transmissivity coefficient (Kt) in 16 municipalities of amazonic region of Mato Grosso State. The data used were obtained by the National Institute of Meteorology, in period between 2008 and 2014. The Kt was obtained by the ratio between global radiation and radiation incident on the top of the atmosphere. It was adopted the sky coverage classification in Kt function for daily partition into four classes: I - cloudy sky $(\mathrm{Kt} \leq 0.35)$, II - partially cloudy sky $(0.35 \leq \mathrm{Kt} \geq 0.55)$, III - partially open sky $(0.55 \leq \mathrm{Kt} \geq 0.65)$ and IV - open sky $(\mathrm{Kt}>0.65)$. Between April to September (dry season) occurs higher indexes of atmospheric transmissivity due to the low attenuation of solar radiation by interaction with atmospheric constituents, with higher frequencies of sky condition IV and II. The sky coverage conditions are dependent on regional rainfall behavior and show differentiated average percentage between the municipalities in the amazonic biome.
\end{abstract}

Keywords: atmospheric absorption, climatic conditions, clearness index, nebulosity.

Autor de correspondência: Adilson Pacheco de Souza, adilsonpacheco@ufmt.br, pachecoufmt@gmail.com. 


\section{Introdução}

A radiação solar constitui-se como uma fonte energética essencial para a existência e manutenção da vida na Terra, estando envolvida em diversos processos físicos, biofísicos e bioquímicos (Neto et al., 2009). Nesse contexto, está relacionada com as áreas de produção agrícola, planejamento urbano, projetos arquitetônicos, análises de poluição atmosférica, estudos micrometeorológicos, energias renováveis, dentre outras (Préndez et al., 1995; Arboit et al., 2008; Stanhill e Cohen, 2001; Oliveira et al., 2002).

Em geral, a radiação solar pode ser considerada como uma das mais importantes fontes de energia renovável (Tiba et al., 2002; Ortega et al., 2010; Varella et al., 2009), pois gera menor impacto ambiental e constitui-se como uma fonte gratuita e abundante de energia, com baixos requisitos de manutenção (Furlan, et al., 2012; Ehnberg e Bollen, 2005).

Por conseguinte, estudos a respeito da sazonalidade e disponibilidade da radiação solar são indispensáveis para o estabelecimento e planejamento estratégico nas áreas supracitadas (Mellit e Kalogirou, 2008; Jebaraj e Iniyanb, 2006; Souza et al., 2010).

Embora tenha-se ciência da importância dos conhecimentos a respeito das variações espaciais e da evolução sazonal da radiação solar incidente na superfície, existem vários fatores que dificultam a obtenção direta das suas componentes espectrais e/ou de atenuação atmosférica (Oliveira et al., 2002). Normalmente, o alto custo relacionado à aquisição e manutenção de equipamentos de medição limita e restringe as medidas de rotina em centros de pesquisa e universidades (Coppolino, 1994; Ampratwum e Dorvolo, 1999), afetando, consequentemente, a obtenção destas informações em extensão continental (Souza et al., 2011).

De acordo com Oliveira et al. (2002), o Brasil possui uma rede solarimétrica muito esparsa, e neste caso, a cobertura limitada das redes de medição de radiação perfazem na necessidade do desenvolvimento e calibração de modelos de estimativa, ao invés dos estudos serem baseados em dados medidos in sito e em longo prazo (Wong e Chow, 2001).

A radiação solar incidente sobre a superfície terrestre varia temporalmente devido a fatores climáticos, geográficos e astronômicos (Codato et al., 2008; Escobedo et al., 2009; Furlan et al., 2012; Teramoto et al., 2012). Contudo, a transmissividade da radiação solar na atmosfera é dependente de fatores da dinâmica atmosférica, envolvendo fatores como pressão do ar, umidade e tipo de nuvem (Nielsen et al., 1981; Albizzati et al., 1997). As nuvens constituem-se como as principais atenuadoras da radiação solar, tendo em vista que absorvem comprimentos de onda específicos (infravermelho) e refletem e difundem (anisotropicamente) grande parte da radiação solar (Iqbal, 1983).

Neste contexto, o coeficiente de transmissividade atmosférica $(\mathrm{Kt})$ refere-se à razão entre as irradiâncias global e atmosférica e permite estabelecer a classificação da cobertura do céu quanto a sua nebulosidade (Iqbal, 1983; Wenxian et al., 1995; Scolar et al., 2003; Codato et al., 2008; Souza et al., 2010).

De acordo com Souza et al. (2012), o Estado de Mato Grosso possui em 33 municípios uma rede de estações meteorológicas automáticas do Instituto Nacional de Meteorologia (INMET) com os objetivos de monitorar e avaliar as variações meteorológicas regionais. As regiões Norte e Noroeste do Estado geralmente apresentam um clima quente e úmido, com a estação de chuva definida entre os meses de outubro a abril, e a seca de maio a setembro e com temperaturas médias anuais se em torno de $24,0{ }^{\circ} \mathrm{C}$. A precipitação anual varia de 1850 a $2400 \mathrm{~mm}$, e a umidade relativa, de 35 a $80 \%$ para os períodos secos e chuvosos, respectivamente. Os padrões de vegetação das mencionadas regiões apresentam características concernentes à Floresta Amazônica, Cerrado e uma faixa de transição Cerrado-Amazônia.

Tendo em vista a extrema importância dos estudos relacionados à radiação solar e a carência de informações deste cunho, o presente trabalho objetivou avaliar as variações das médias mensais da radiação solar e as frequências da transmissividade atmosférica global para 16 estações meteorológicas automáticas inseridas no bioma amazônico do estado de Mato Grosso.

\section{Material e Métodos}

Os dados de radiação solar utilizados foram disponibilizados pelo Instituto Nacional de Meteorologia (INMET), o qual possui uma rede de estações meteorológicas automáticas cujos valores dos elementos meteorológicos são observados a cada minuto e armazenados em valores horários.

Analisou-se a base de dados de dezesseis estações automáticas localizadas na região amazônica do Estado de Mato Grosso (Fig. 1). Os valores discrepantes foram removidos das séries, assim como os valores de coeficiente de transmissividade atmosférica $(\mathrm{Kt})$ acima de 0,81 , pelo fato de não ocorrer uma sequência lógica de evolução das três radiações em função da transmissividade (Escobedo et al., 2009). Na Tabela 1 encontram-se descritos os períodos de dados analisados e as porcentagens de perdas referentes aos valores de radiação solar para cada município.

A porcentagem de perdas decorreu da análise de consistência da base de dados e da eliminação dos dias com erros de medição. Foram obtidas as médias mensais diárias da radiação global e do coeficiente de transmissividade para cada município, assim como a média mensal diária de Kt para a região. A transmissividade atmosférica foi obtida através da razão entre a radiação global e a radiação incidente no topo da atmosfera, dada pela integração entre o nascer e o pôr do sol dos valores da radiação incidente no topo da atmosfera na partição horária, que por sua vez, foi estimada pela equação de Iqbal (1983). 


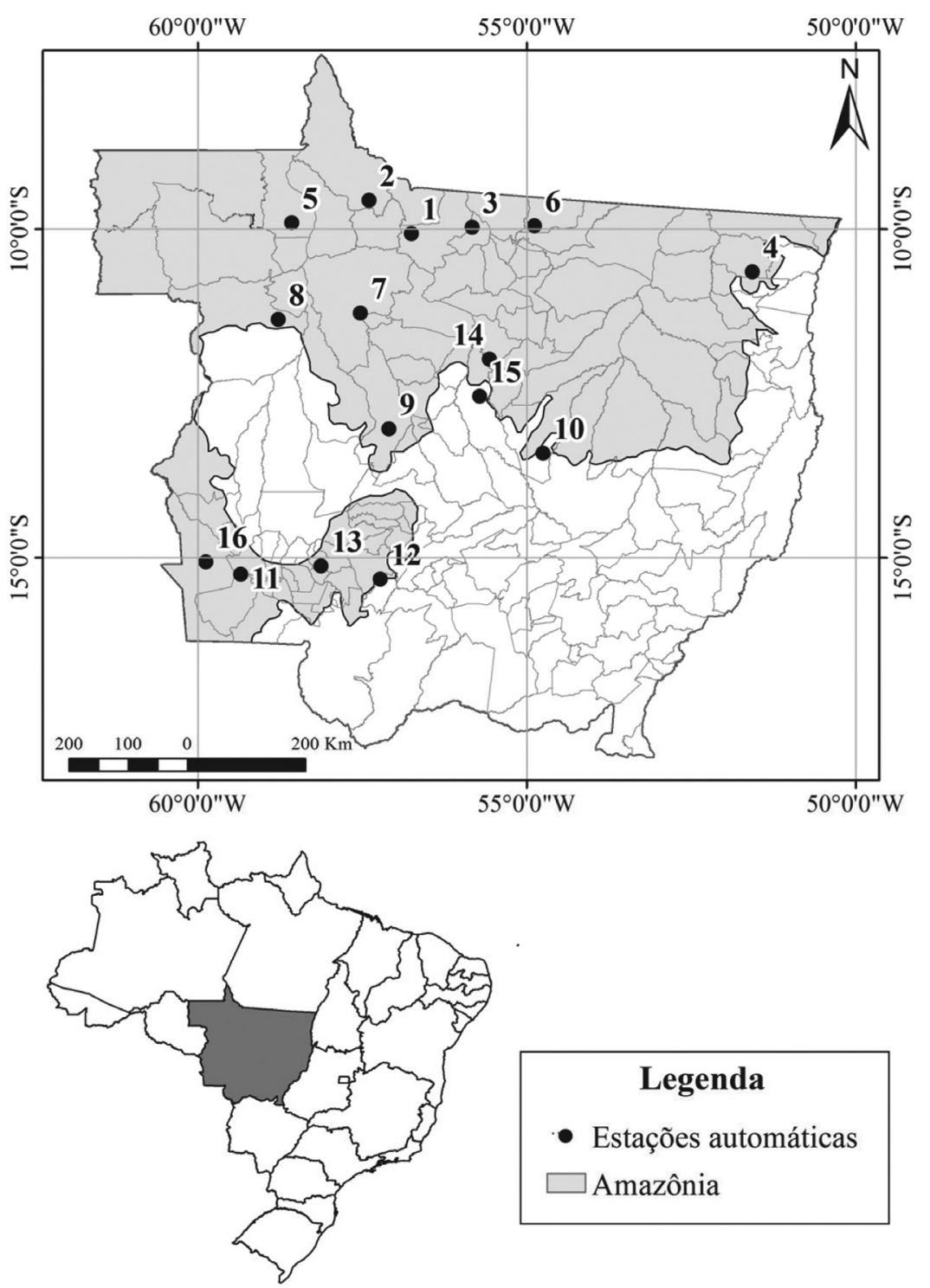

Figura 1 - Mapa de localização das estações meteorológicas automáticas da rede do INMET instaladas na região amazônica do estado de Mato Grosso.

Conforme os dados médios mensais diários de $\mathrm{Kt}$ estabeleceu-se a classificação da cobertura de céu para cada estação automática, em função do coeficiente de transmissividade proposto por Escobedo et al. (2009). Esta metodologia dispensa o uso de dados de radiação direta e/ou difusa, e considera os seguintes tipos de condição de céu para a partição diária: I, quando $\mathrm{Kt} \leq 0,35$, a radiação direta é praticamente nula, sendo a radiação global igual à radiação difusa (céu nublado); II, se $0,35 \leq \mathrm{Kt} \geq 0,55$, a radiação global é composta principalmente pela fração difusa e em menor escala pela radiação direta (céu parcialmente nublado); III, se $0,55 \leq \mathrm{Kt} \geq 0,65$, é o inverso do segundo intervalo, visto que a radiação difusa diminui gradativamente (céu parcialmente aberto); e IV, quando Kt >0,65, a radiação global possui a maior parcela composta pela radiação direta e mínima difusa, portanto, a cobertura do céu é considerada aberta. Em seguida, realizou-se a distribuição de frequência da base de dados de cada município por condição de cobertura de céu.

\section{Resultados e Discussão}

Nas Figs. 2 e 3 pode-se observar a evolução da radiação global ao longo do período estudado para cada município, respectivamente. Nas Tabelas 2 e 3 encontram-se descritos os valores médios mensais diários e desvios por município para a radiação global e Kt, respectivamente. $\mathrm{Na}$ Fig. 3 são apresentados os valores médios mensais diários e desvios de Kt para a região. Os desvios médios mensais de Kt para cada município em relação à média mensal da região são apresentados na Fig. 4.

Através da variação existente entre os valores médios mensais diários de Kt ao longo do ano é possível observar 
Tabela 1 - Informações das estações meteorológicas automáticas instaladas na região amazônica do Estado de Mato Grosso.

\begin{tabular}{|c|c|c|c|c|c|c|c|}
\hline Cód. & Nome da Estação (Município) & Latitude & Longitude & Altitude (m) & Período de dados & Número de dados & Perdas $(\%)$ \\
\hline A-924 & 1. Alta Floresta & $-10,0672$ & $-56,7522$ & 294 & $09 / 2011-12 / 2014$ & 1217 & 10,93 \\
\hline A-910 & 2. Apiacás & $-9,5639$ & $-57,3936$ & 220 & $01 / 2008-12 / 2014$ & 2556 & 21,48 \\
\hline A-926 & 3. Carlinda & $-9,9703$ & $-55,8272$ & 300 & $04 / 2008-12 / 2014$ & 2465 & 10,43 \\
\hline A-918 & 4. Confresa & $-10,6539$ & $-51,5668$ & 237 & $06 / 2008-12 / 2014$ & 2404 & 15,60 \\
\hline A-919 & 5. Cotriguaçu & $-9,9061$ & $-58,5719$ & 261 & $01 / 2008-12 / 2014$ & 2556 & 12,75 \\
\hline A-906 & 6. Guarantã do Norte & $-9,9500$ & $-54,8833$ & 320 & $01 / 2008-12 / 2014$ & 2556 & 18,15 \\
\hline A-920 & 8. Juína & $-11,375$ & $-58,775$ & 374 & $01 / 2009-12 / 2012$ & 2190 & 43,06 \\
\hline A-928 & 9. Nova Maringá & $-13,0386$ & $-57,0922$ & 353 & $04 / 2008-12 / 2014$ & 2465 & 18,66 \\
\hline A-929 & 10. Nova Ubiratã & $-13,4111$ & $-54,7522$ & 518 & $04 / 2008-12 / 2014$ & 2465 & 42,31 \\
\hline A-917 & 11. Pontes e Lacerda & $-15,2511$ & $-59,3467$ & 256 & $01 / 2008-12 / 2014$ & 2556 & 17,53 \\
\hline A-935 & 12. Porto Estrela & $-15,3247$ & $-57,2264$ & 145 & $02 / 2008-12 / 2014$ & 2525 & 41,50 \\
\hline A-936 & 13. Salto do Céu & $-15,1247$ & $-58,1275$ & 303 & $01 / 2008-12 / 2014$ & 2556 & 10,84 \\
\hline A-917 & 14. Sinop & $-11,9822$ & $-55,5658$ & 371 & $01 / 2008-02 / 2014$ & 2253 & 42,74 \\
\hline A-922 & 16. Vila Bela da Santíssima Trindade & $-15,0628$ & $-59,8729$ & 222 & $01 / 2008-02 / 2014$ & 2253 & 18,69 \\
\hline
\end{tabular}
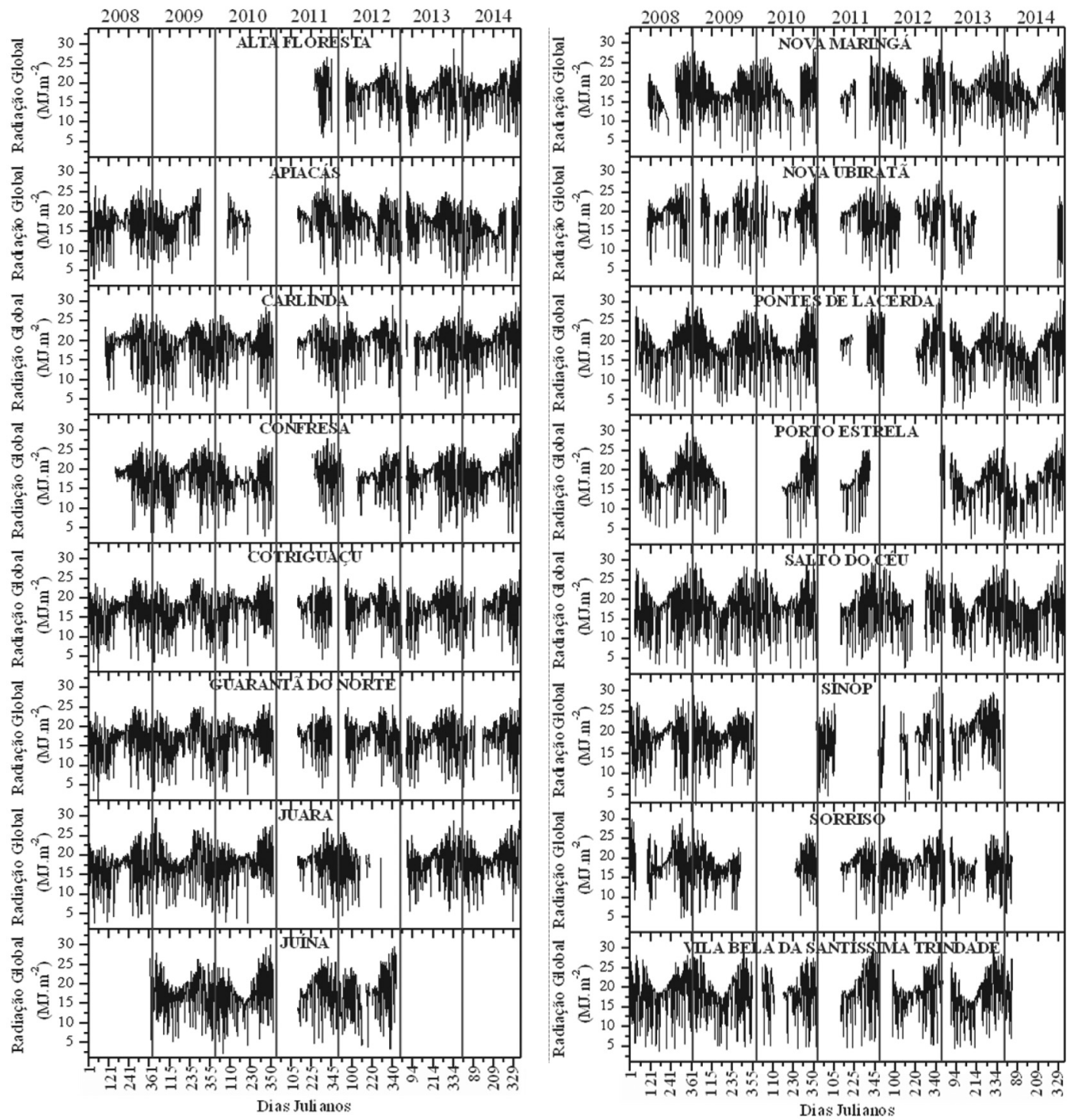

Figura 2 - Evolução diária da radiação global ( $\mathrm{MJ} \mathrm{m}^{-2}$ dia $\left.^{-1}\right)$ entre 01/2008 e 12/2014, para dezesseis estações meteorológicas automáticas instaladas na região amazônica do Estado de Mato Grosso. 

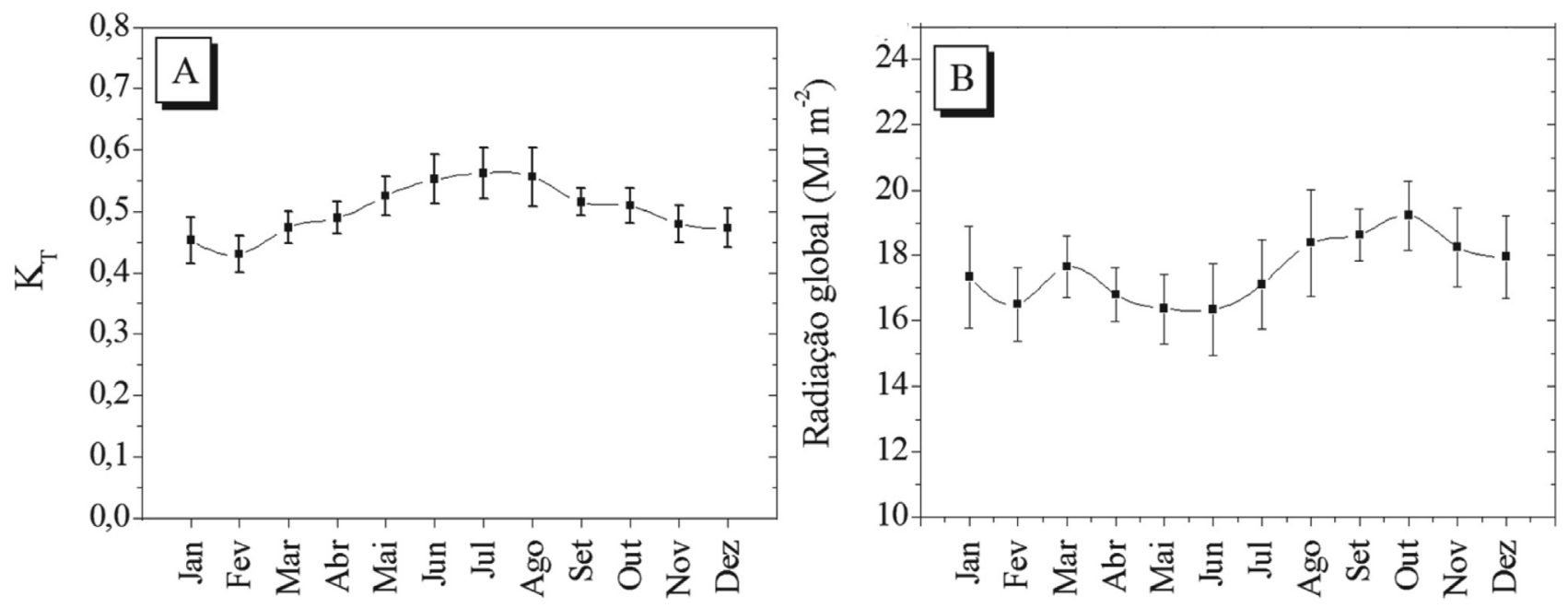

Figura 3 - Valores médios mensais da radiação global diária $\left(\mathrm{MJ} \mathrm{m}^{-2} \mathrm{dia}^{-1}\right)$ e do coeficiente de transmissividade atmosférica $\left(\mathrm{K}_{\mathrm{T}}\right)$ para a região amazônica do Mato Grosso.

Tabela 2 - Médias mensais e desvio padrão (entre parenteses) da radiação global diária ( $\left.\mathrm{MJ} \mathrm{m}^{-2} \operatorname{~ia}^{-1}\right)$ para dezesseis estações meteorológicas automáticas instaladas na região amazônica do Estado de Mato Grosso.

\begin{tabular}{|c|c|c|c|c|c|c|c|c|}
\hline \multicolumn{9}{|c|}{ Estações meteorológicas automáticas (municípios) } \\
\hline Mês & Alta Floresta & Apiacás & Carlinda & Confresa & Cotriguaçu & Guaranta do Norte & Juara & Juína \\
\hline Jan & $15,58(1,85)$ & $15,44(1,69)$ & $17,27(1,65)$ & $17,54(0,79)$ & $14,81(0,80)$ & $14,61(1,68)$ & $17,83(2,09)$ & $16,93(1,33)$ \\
\hline Fev & $15,07(0,03)$ & $16,01(2,55)$ & $17,24(0,17)$ & $17,21(0,95)$ & $14,58(1,73)$ & $15,55(3,33)$ & $17,00(1,46)$ & $16,50(1,51)$ \\
\hline Mar & $16,61(0,60)$ & $17,03(1,37)$ & $19,60(1,74)$ & $17,47(0,60)$ & $15,61(1,24)$ & $17,88(1,73)$ & $17,30(0,93)$ & $17,41(1,06)$ \\
\hline Abr & $15,81(1,38)$ & $16,69(1,83)$ & $17,62(1,60)$ & $15,80(1,28)$ & $16,12(1,35)$ & $18,17(1,14)$ & $16,47(0,88)$ & $16,92(0,87)$ \\
\hline Mai & $16,19(0,47)$ & $17,07(1,74)$ & $18,00(0,98)$ & $16,35(0,94)$ & $16,33(1,75)$ & $16,45(1,84)$ & $16,79(1,24)$ & $15,44(1,43)$ \\
\hline Jun & $16,65(0,90)$ & $17,74(1,08)$ & $18,84(0,72)$ & $17,87(0,72)$ & $17,14(0,78)$ & $15,54(1,20)$ & $17,21(0,87)$ & $13,56(4,00)$ \\
\hline Jul & $17,89(0,66)$ & $17,30(1,48)$ & $19,83(0,69)$ & $18,43(1,12)$ & $17,89(0,53)$ & $15,21(1,24)$ & $18,10(0,48)$ & $16,60(1,68)$ \\
\hline Ago & $19,04(0,51)$ & $16,82(1,83)$ & $20,65(0,97)$ & $19,64(1,78)$ & $18,68(0,90)$ & $15,92(2,91)$ & $18,82(1,07)$ & $17,61(1,30)$ \\
\hline Set & $19,20(1,20)$ & $17,95(1,75)$ & $19,86(0,81)$ & $18,29(2,08)$ & $18,21(0,61)$ & $17,36(1,96)$ & $18,13(1,91)$ & $18,42(1,25)$ \\
\hline Out & $18,84(1,25)$ & $18,35(1,65)$ & $19,94(0,82)$ & $18,66(0,60)$ & $18,59(0,87)$ & $16,59(1,37)$ & $19,72(1,16)$ & $20,34(0,85)$ \\
\hline Nov & $17,59(1,50)$ & $17,22(2,29)$ & $18,57(1,33)$ & $18,45(0,85)$ & $16,91(0,62)$ & $15,48(1,39)$ & $18,63(1,06)$ & $18,47(1,37)$ \\
\hline Dez & $17,54(0,86)$ & $16,54(1,23)$ & $17,79(1,57)$ & $18,46(2,17)$ & $16,40(1,49)$ & $15,18(1,94)$ & $17,94(1,38)$ & $18,80(3,14)$ \\
\hline Mês & Nova Maringá & Nova Ubiratã & Pontes e Lacerda & Porto Estrela & Salto do Céu & Sinop & Sorriso & Vila Bela S. Trindade \\
\hline Jan & $17,83(1,76)$ & $17,58(1,17)$ & $19,25(1,44)$ & $19,29(2,59)$ & $17,59(1,92)$ & $18,34(1,66)$ & $17,73(2,04)$ & $19,81(1,65)$ \\
\hline Fev & $16,03(2,81)$ & $18,97(1,29)$ & $16,96(2,40)$ & $15,51(2,27)$ & $16,33(2,88)$ & $16,56(2,82)$ & $16,41(1,66)$ & $18,23(1,07)$ \\
\hline Mar & $17,38(0,84)$ & $18,61(0,88)$ & $17,88(1,36)$ & $17,04(2,87)$ & $17,28(0,69)$ & $18,30(0,81)$ & $18,69(0,32)$ & $18,32(0,61)$ \\
\hline Abr & $17,23(0,70)$ & $17,95(1,71)$ & $17,22(0,90)$ & $15,08(3,69)$ & $16,65(0,88)$ & $17,27(0,75)$ & $16,98(1,25)$ & $17,07(1,18)$ \\
\hline Mai & $16,02(0,71)$ & $17,31(2,04)$ & $15,79(1,96)$ & $14,59(1,51)$ & $15,10(0,96)$ & $18,56(1,04)$ & $16,91(0,71)$ & $15,17(0,95)$ \\
\hline Jun & $15,13(1,05)$ & $16,92(2,40)$ & $15,41(2,17)$ & $14,72(0,55)$ & $14,97(1,25)$ & $17,39(2,34)$ & $16,62(0,57)$ & $15,73(1,65)$ \\
\hline Jul & $15,46(1,61)$ & $18,88(0,77)$ & $16,38(1,78)$ & $15,21(2,04)$ & $15,86(1,32)$ & $16,36(6,79)$ & $17,70(0,62)$ & $16,79(1,31)$ \\
\hline Ago & $15,88(3,31)$ & $20,63(1,11)$ & $18,10(1,61)$ & $16,43(1,20)$ & $18,06(0,86)$ & $21,26(1,73)$ & $18,55(1,11)$ & $18,22(0,80)$ \\
\hline Set & $19,40(1,39)$ & $19,85(0,52)$ & $18,39(0,77)$ & $17,68(1,11)$ & $17,83(0,93)$ & $19,72(1,20)$ & $19,08(2,00)$ & $18,88(0,82)$ \\
\hline Out & $19,36(0,89)$ & $19,57(0,91)$ & $19,62(0,62)$ & $19,04(0,43)$ & $18,50(0,99)$ & $21,17(0,83)$ & $18,97(1,42)$ & $20,40(0,54)$ \\
\hline Nov & $18,14(1,28)$ & $17,81(2,58)$ & $19,91(1,17)$ & $20,13(1,26)$ & $18,67(1,59)$ & $18,13(0,99)$ & $17,88(1,10)$ & $20,17(1,97)$ \\
\hline Dez & $18,23(1,54)$ & $18,10(3,11)$ & $20,27(1,42)$ & $19,16(0,93)$ & $17,96(0,99)$ & $18,18(0,99)$ & $17,11(1,34)$ & $19,77(1,50)$ \\
\hline
\end{tabular}


Tabela 3 - Médias mensais e desvio padrão (entre parenteses) dos valores diários do coeficiente de transmissividade atmosférica $\left(\mathrm{K}_{\mathrm{T}}\right)$ para dezesseis estações meteorológicas automáticas instaladas na região amazônica do Estado de Mato Grosso.

\begin{tabular}{|c|c|c|c|c|c|c|c|c|}
\hline \multicolumn{9}{|c|}{ Estações meteorológicas automáticas (municípios) } \\
\hline Mês & Alta Floresta & Apiacás & Carlinda & Confresa & Cotriguaçu & Guarantã do Norte & Juara & Juína \\
\hline Jan & $0,41(0,05)$ & $0,41(0,04)$ & $0,45(0,04)$ & $0,46(0,02)$ & $0,39(0,02)$ & $0,38(0,04)$ & $0,47(0,05)$ & $0,44(0,04)$ \\
\hline Fev & $0,39(0,01)$ & $0,42(0,07)$ & $0,45(0,01)$ & $0,45(0,02)$ & $0,38(0,05)$ & $0,41(0,09)$ & $0,44(0,04)$ & $0,43(0,04)$ \\
\hline Mar & $0,44(0,02)$ & $0,45(0,04)$ & $0,52(0,04)$ & $0,47(0,02)$ & $0,42(0,03)$ & $0,48(0,05)$ & $0,46(0,02)$ & $0,47(0,03)$ \\
\hline Abr & $0,45(0,04)$ & $0,48(0,05)$ & $0,51(0,04)$ & $0,45(0,04)$ & $0,46(0,04)$ & $0,52(0,03)$ & $0,48(0,03)$ & $0,49(0,03)$ \\
\hline Mai & $0,51(0,02)$ & $0,53(0,06)$ & $0,56(0,03)$ & $0,52(0,03)$ & $0,51(0,06)$ & $0,51(0,06)$ & $0,53(0,05)$ & $0,47(0,07)$ \\
\hline Jun & $0,55(0,03)$ & $0,58(0,04)$ & $0,62(0,02)$ & $0,59(0,02)$ & $0,56(0,03)$ & $0,51(0,04)$ & $0,58(0,03)$ & $0,45(0,13)$ \\
\hline Jul & $0,57(0,02)$ & $0,55(0,05)$ & $0,64(0,02)$ & $0,60(0,04)$ & $0,57(0,02)$ & $0,49(0,04)$ & $0,59(0,02)$ & $0,54(0,05)$ \\
\hline Ago & $0,56(0,01)$ & $0,50(0,05)$ & $0,61(0,03)$ & $0,58(0,05)$ & $0,55(0,03)$ & $0,47(0,09)$ & $0,57(0,03)$ & $0,53(0,04)$ \\
\hline Set & $0,53(0,03)$ & $0,49(0,05)$ & $0,54(0,02)$ & $0,50(0,06)$ & $0,50(0,02)$ & $0,48(0,05)$ & $0,50(0,06)$ & $0,51(0,03)$ \\
\hline Out & $0,50(0,03)$ & $0,49(0,04)$ & $0,53(0,02)$ & $0,49(0,02)$ & $0,49(0,02)$ & $0,44(0,04)$ & $0,52(0,03)$ & $0,54(0,02)$ \\
\hline Nov & $0,46(0,04)$ & $0,46(0,06)$ & $0,49(0,04)$ & $0,49(0,02)$ & $0,45(0,02)$ & $0,41(0,04)$ & $0,49(0,03)$ & $0,49(0,04)$ \\
\hline Dez & $0,47(0,02)$ & $0,44(0,03)$ & $0,47(0,04)$ & $0,49(0,06)$ & $0,44(0,04)$ & $0,40(0,05)$ & $0,47(0,04)$ & $0,50(0,08)$ \\
\hline Mês & Nova Maringá & Nova Ubiratã & Pontes e Lacerda & Porto Estrela & Salto do Céu & Sinop & Sorriso & Vila Bela S.Trindade \\
\hline Jan & $0,47(0,05)$ & $0,46(0,03)$ & $0,50(0,04)$ & $0,50(0,07)$ & $0,46(0,05)$ & $0,48(0,04)$ & $0,46(0,05)$ & $0,50(0,06)$ \\
\hline Fev & $0,42(0,07)$ & $0,50(0,03)$ & $0,44(0,06)$ & $0,41(0,06)$ & $0,43(0,07)$ & $0,43(0,07)$ & $0,43(0,04)$ & $0,48(0,03)$ \\
\hline Mar & $0,47(0,02)$ & $0,50(0,02)$ & $0,49(0,04)$ & $0,46(0,08)$ & $0,47(0,02)$ & $0,49(0,02)$ & $0,50(0,01)$ & $0,51(0,01)$ \\
\hline Abr & $0,51(0,02)$ & $0,53(0,06)$ & $0,51(0,03)$ & $0,45(0,11)$ & $0,49(0,03)$ & $0,50(0,02)$ & $0,50(0,03)$ & $0,51(0,03)$ \\
\hline Mai & $0,52(0,02)$ & $0,57(0,07)$ & $0,53(0,07)$ & $0,49(0,05)$ & $0,50(0,03)$ & $0,59(0,03)$ & $0,54(0,02)$ & $0,51(0,04)$ \\
\hline Jun & $0,52(0,04)$ & $0,58(0,08)$ & $0,54(0,08)$ & $0,52(0,02)$ & $0,53(0,04)$ & $0,59(0,08)$ & $0,56(0,02)$ & $0,55(0,06)$ \\
\hline Jul & $0,51(0,05)$ & $0,63(0,02)$ & $0,56(0,06)$ & $0,50(0,03)$ & $0,54(0,05)$ & $0,54(0,22)$ & $0,58(0,02)$ & $0,57(0,04)$ \\
\hline Ago & $0,49(0,10)$ & $0,62(0,03)$ & $0,57(0,06)$ & $0,51(0,04)$ & $0,56(0,02)$ & $0,64(0,05)$ & $0,56(0,04)$ & $0,57(0,02)$ \\
\hline Set & $0,53(0,04)$ & $0,55(0,01)$ & $0,52(0,02)$ & $0,50(0,03)$ & $0,50(0,03)$ & $0,54(0,03)$ & $0,53(0,05)$ & $0,53(0,02)$ \\
\hline Out & $0,51(0,02)$ & $0,52(0,02)$ & $0,52(0,02)$ & $0,51(0,01)$ & $0,49(0,03)$ & $0,56(0,02)$ & $0,50(0,04)$ & $0,54(0,02)$ \\
\hline Nov & $0,48(0,03)$ & $0,47(0,07)$ & $0,52(0,03)$ & $0,53(0,03)$ & $0,49(0,04)$ & $0,48(0,05)$ & $0,47(0,03)$ & $0,53(0,05)$ \\
\hline Dez & $0,48(0,04)$ & $0,48(0,08)$ & $0,53(0,04)$ & $0,50(0,02)$ & $0,47(0,03)$ & $0,48(0,03)$ & $0,45(0,04)$ & $0,51(0,04)$ \\
\hline
\end{tabular}

como a composição atmosférica (nuvens, vapor de água e aerossóis) interfere na transmissão da radiação global.

Os meses de abril a setembro, correspondentes à estação seca na região estudada, apresentam os maiores índices de transmissividade atmosférica para a região. Isso se deve à baixa atenuação da radiação solar pela interação com os constituintes atmosféricos. Já durante a estação chuvosa, em geral, os valores de Kt são menores, tendo em vista que nesta época a concentração de nuvens e vapor d'água na atmosfera é a maior do ano.

Porém, durante o período de seca alguns municípios apresentam valores baixos de transmissividade atmosférica em relação à média da região estudada, dados que podem ser explicados pela influência dos aerossóis presentes na atmosfera, devido principalmente às queimadas comuns nesta época do ano.

Os municípios de Confresa, Cotriguaçu, Juara, Juína, Nova Maringá e Nova Ubiratã fazem parte da Lista de Municípios Prioritários da Amazônia, nos quais são destacadas as medidas de integração e aperfeiçoamento das ações de monitoramento e controle de órgãos federais, o ordenamento fundiário e territorial e o incentivo a atividades econômicas ambientalmente sustentáveis. Tais municípios integram a lista devido à grande área de floresta desmatada anualmente (MMA, 2013).

Nos meses de julho e agosto, os municípios de Guarantã do Norte e Nova Maringá apresentam médias mensais de $\mathrm{Kt}$ abaixo dos valores médios da região para a mesma época do ano. Através da análise do banco de dados de Nova Maringá, observam-se valores discrepantes de Kt para tais meses no ano de 2008, no qual o município apresentou 479 focos de calor, ocupando a terceira posição dentre os municípios do Estado com maior quantidade de registros, sendo que somente entre os meses de julho e outubro foram totalizados mais de 30.000 ha de área queimada (SEMA, 2010; Machado e Alves, 2011).

A estação de Guarantã do Norte apresenta os menores valores de Kt para os meses mencionados anteriormente, nos anos de 2013 e 2014, nos quais foram registrados 105 e 43 focos de calor dentre julho e agosto, respectivamente. 


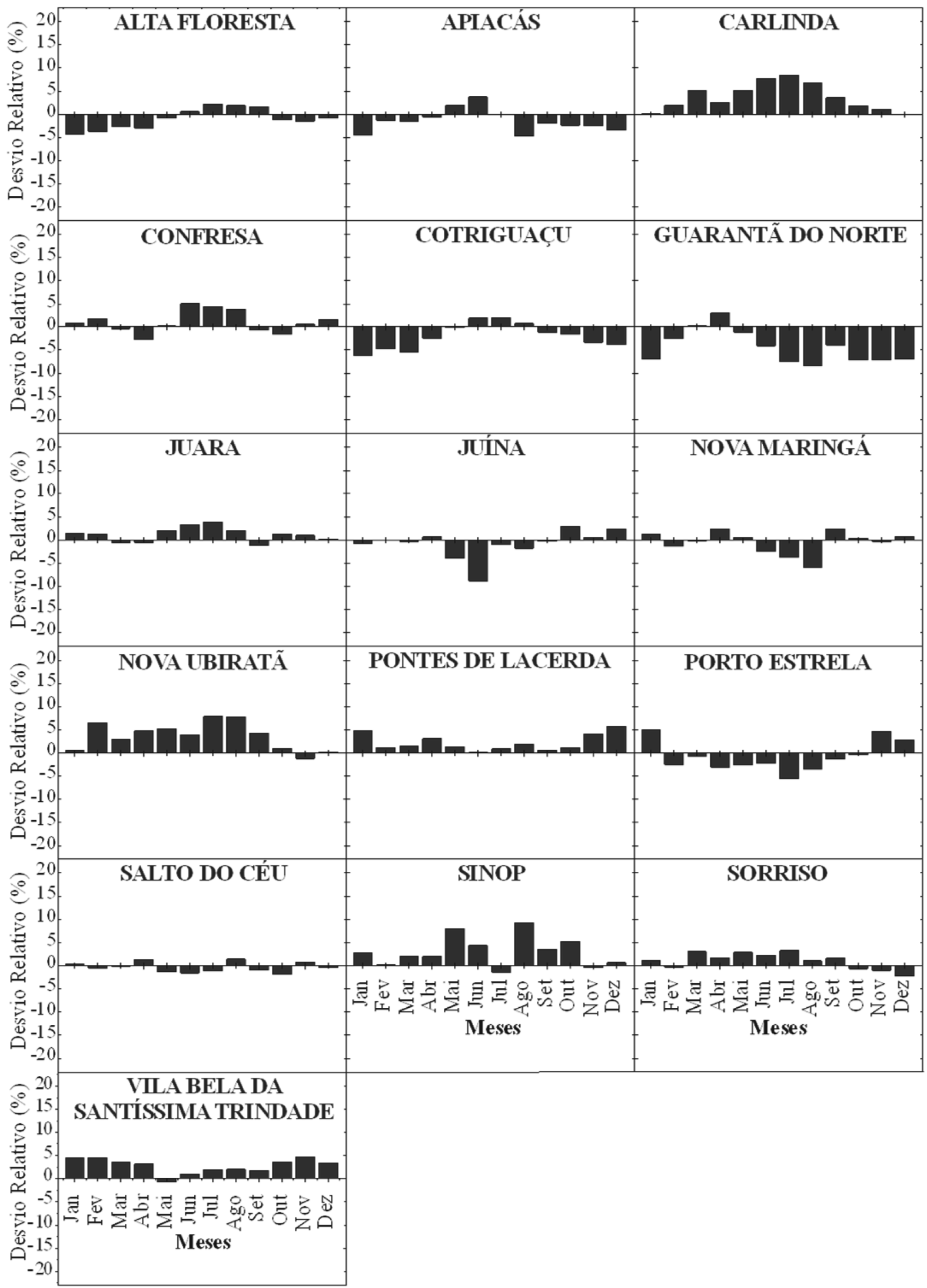

Figura 4 - Desvios médios mensais de $\mathrm{K}_{\mathrm{T}}$ em relação à média mensal da região. 


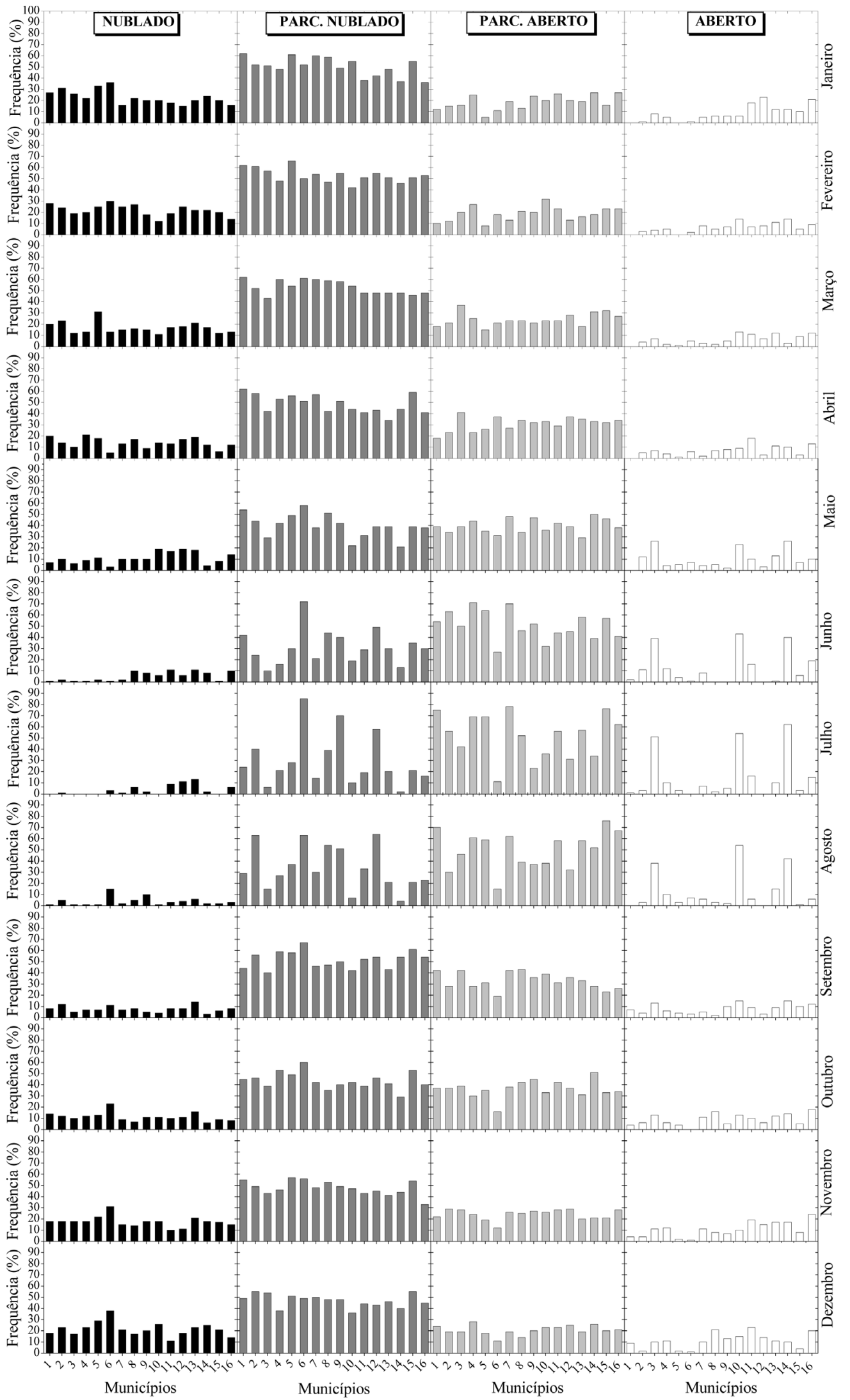

Figura 5 - Evolução anual da frequência diária mensal das coberturas de céu (em função do $\mathrm{K}_{\mathrm{T}}$ ) para cada município (Numeração do eixo "X": 1. Alta Floresta; 2. Apiacás; 3. Carlinda; 4. Confresa; 5. Cotriguaçu; 6. Guarantã do Norte; 7. Juara; 8. Juína; 9. Nova Maringá; 10. Nova Ubiratã; 11. Pontes e Lacerda; 12. Porto Estrela; 13. Salto do Céu; 14. Sinop; 15. Sorriso; 16. Vila Bela da Santí́ssima Trindade). 
Por fazer divisa com os municípios de Altamira e Novo Progresso - PA, que apresentam elevadas taxas de desmatamento (MMA, 2013), Guarantã também fica sujeita à recepção de aerossóis de queimadas provenientes desta região, visto que tais partículas podem ser transportadas por até milhares de quilômetros dos locais de emissões.

Os maiores valores médios mensais de Kt durante a estação seca são encontrados para o município de Sinop, enquanto que os menores valores médios mensais referentes à estação chuvosa são encontrados para Cotriguaçu. Esses dados podem ser relacionados ao índice pluviométrico dos municípios durante as estações do ano.

Na Fig. 5 são apresentadas as frequências de coberturas de céu ao longo do ano, sendo que no período seco ocorrem maiores frequência de condição de céu aberto, enquanto que no restante do ano predomina a condição de céu parcialmente nublado.

A frequência de dias com condição de céu I (céu nublado) para a região amazônica de Mato Grosso é maior durante o período chuvoso do ano, corroborando com Teramoto e Escobedo (2012) em análises de frequência de coberturas de céu para Botucatu-SP.

$\mathrm{O}$ mês de janeiro apresentou a maior frequência de dias com cobertura de céu I $(24,84 \%)$ e julho a menor $(0,44 \%)$. O oposto é observado para a cobertura de céu aberto (IV), para a qual a frequência é maior durante o período seco do ano, devido à predominância de condições atmosféricas de baixa nebulosidade e concentração de vapor d'água (Teramoto e Escobedo, 2012). A maior frequência de dias com cobertura de céu IV (aberto) foi observada em julho $(38,16 \%)$ e a menor em janeiro $(4,94 \%)$.

Em relação à cobertura de céu II (parcialmente nublado), os maiores valores de frequência são observados durante os meses chuvosos, similar à variação da cobertura de céu I. Para a cobertura de céu III, os valores de frequência variaram de $42,49 \%$ em junho a $15,84 \%$ em janeiro.

De acordo com Querino et al. (2010), o fenômeno de precipitação também deve ser levado em consideração durante a análise das condições de céu, pois além de influenciar vários processos na superfície da Terra é responsável também pela limpeza da atmosfera, visto que no ato da precipitação grande parte das partículas em suspensão retornam para a superfície deixando a atmosfera mais limpa.

\section{Conclusões}

O comportamento das condições de cobertura de céu ao longo do ano e entre os municípios da região amazônica do estado de Mato Grosso é dependente do comportamento pluviométrico regional e das modificações na composição da atmosfera.

A radiação global média diária da região amazônica do estado de Mato Grosso varia de 16,34 $\pm 1,39$ (junho) a $19,22 \pm 1,05 \mathrm{MJ} \mathrm{m}^{-2} \mathrm{dia}^{-1}$ (outubro) e os coeficientes de transmissividade atmosférica $(\mathrm{Kt})$ variam de $0,56 \pm 0,04$ (julho) a $0,43 \pm 0,03$ (fevereiro).

\section{Agradecimentos}

Ao Instituto Nacional de Meteorologia (INMET) pela disponibilização das bases de dados meteorológicos históricos inseridos no Banco de Dados Meteorológicos para Ensino e Pesquisa (BDMEP), e à Coordenação de Aperfeiçoamento de Pessoal de Nível Superior (Capes) e Fundação de Amparo à Pesquisa no Estado de Mato Grosso (FAPEMAT) pela concessão de bolsas de mestrado.

\section{Referências}

ALBIZZATI, E.; ROSSETTI, G.; ALFANO, O. Measurements and predictions of solar radiation incident on horizontal surfaces at Santa Fe, Argentina (3139'S, 6043'W). Renewable Energy, v. 11, n. 4, p. 469-478, 1997.

AMPRATWUM, D.B.; DORVLO, A.S.S. Estimation of solar radiation from the number of sunshine hours. Applied Energy, v. 63, p. 161-167, 1999.

ARBOIT, M.; DIBLASI, A.; FERNANDEZ LLANO, J.C.; DE ROSA, C. Assessing the solar potential of low-density urban environments in Andean cities with desert climates: the case of the city of Mendoza, in Argentina. Renewable Energy, v. 33, p. 1733-1748, 2008.

CODATO, G.; OLIVEIRA, A.P.; SOARES, J.; ESCOBEDO, J.F.; GOMES, E.N.; et al. Global and diffuse solar irradiance in urban and rural areas in Southeast Brazil. Theoretical and Applied Climatology, v. 93, n. 1, p. 57-73, 2008.

COPPOLINO, S. A new correlation between clearness index and relative sunshine. Renewable Energy, v. 4, n. 4, p. 417-23, 1994.

EHNBERG, J.S.G.; BOLLEN, M.H.J. Simulation of global solar radiation based on cloud observations. Solar Energy, v. 78, p. 157-162, 2005.

ESCOBEDO, J.F.; GOMES, E.N.; OLIVEIRA, A.P. de; SOARES, J. Modeling hourly and daily fractions of UV, PAT and NIR to global solar radiation under various Sky conditions at Botucatu, Brazil. Applied Energy, v. 86, n. 3, p. 299-309, 2009.

FURLAN, C.; OLIVEIRA, A.P. de; SOARES, J.; CODADO, G.; ESCOBEDO, J.F. The role of clouds in improving the regression model for hourly values of diffuse solar radiation. Applied Energy, v. 92, p. 240-254, 2012.

IQBAL, M. An introduction to solar radiation. London: Academic Press. 390p., 1983.

JEBARAJ, S.; INIYANB, S. A review of energy models. Renewable and Sustainable Energy Reviews, v. 10, p. 281-311, 2006.

MACHADO, A.T.; ALVES, M.C. Análise e quantificação de focos de calor utilizando satélites NOAA-12 e NOAA-15 no estado de Mato Grosso. In: Simpósio Brasileiro de Sensoriamento Remoto (SBSR), 15, 2011, Curitiba. Anais... São José dos Campos: INPE: 2011. Artigos, p.7888. Disponível em: http://www.dsr.inpe.br/sbsr2011/files/p1299.pdf . Acesso em: 30 ago. 2012. 
MELLIT, A.; KALOGIROU, S.A. Artificial intelligence techniques for photovoltaic applications: a review. Progress in Energy and Combustion Science, v. 34, p. 574-632, 2008.

MINISTÉRIO DO MEIO AMBIENTE. Lista de Municípios Prioritários da Amazônia. 2013. Disponível em: http://www.mma.gov.br/images/arquivos/florestas/controle_e_prevencao/2014/lista $\% 20$ municipios_\%20prioritarios_07.08.pdf . Acesso em: 30 nov. 2015.

NETO, O.L.M.; COSTA, A.A.; RAMALHO, F.P. Estimativa de radiação solar via modelagem atmosférica de mesoescala aplicada à região Nordeste do Brasil. Revista Brasileira de Meteorologia, v. 24, n. 3, p. 339-345, 2009.

NIELSEN, L.; PRAHM, L.P.; BERKOWICZ, R.; CONRADSEN, K. Net incoming radiation estimated from hourly global radiation and/or cloud observations. International Journal of Climatology, v. 1, p. 255-272, 1981.

OLIVEIRA, A.P.; ESCOBEDO, J.F.; MACHADO, A.J.; SOARES, J. Correlation models of diffuse solar-radiation applied to the city of São Paulo, Brazil. Applied Energy, v. 71, p. 59-73, 2002.

ORTEGA, A.; ESCOBAR, R.; COLLE, S.; ABREU, S.L. de. The state of solar energy resource assessment in Chile. Renewable Energy, v. 35, p. 2514-24, 2010.

PRENDÉZ, M.M.; EGIDO, M.; TOMAS, C.; SECO, J.; CALVO, A. Correlation between solar radiation and total suspended particulate matter in Santiago, Chile-Preliminary results. Atmospheric Environment, v. 29, n. 13, p. 1543-51, 1995.

QUERINO, C.A.S.; MOURA, M.A.L.; QUERINO, J.K.A.S.; VON RADOW, C.; MARQUES FILHO, A.O. Estudo da radiação solar global e do índice de transmissividade $\left(\mathrm{K}_{\mathrm{T}}\right)$, externo e interno, em uma floresta de mangue em Alagoas Brasil. Revista Brasileira de Meteorologia, v. 26, n. 2, p. 204-294, 2011.

SCOLAR, J.; MARTINS, D.; ESCOBEDO, J.F. Estimativa da irradiação total sobre uma superfície inclinada a partir da irradiação global na horizontal. Revista Brasileira de Geofísica, v. 21, n. 3, p. 249-258, 2003.

SECRETARIA DE ESTADO DO MEIO AMBIENTE - SEMA MATO GROSSO, Superintendência de Monitoramento de
Indicadores Ambientais. Relatório de Monitoramento de Queimadas - 2008 e 2009. Cuiabá: SEMA/MT, 2010.

SOUZA, A.P.; CASAVECCHIA, B.H.; STANGERLIN, D.M. Avaliação dos riscos de incêndios florestais nas regiões Norte e Noroeste da Amazônia Matogrossense. Scientia Plena, v. 8, n. 5, p. 1-14, 2012.

SOUZA, A.P.; ESCOBEDO, F.J.; DAL PAI, A.; GOMES, E.N. Estimativa da radiação global incidente em superfícies inclinadas por modelos isotrópicos e índice de claridade. Pesquisa Agropecuária Brasileira, v. 54, n. 4, p. 339-350, 2010.

SOUZA, A P.; ESCOBEDO, F.J.; DAL PAI, A.; GOMES, E.N. Estimativas das componentes da radiação solar incidente em superfícies inclinadas baseadas na radiação global horizontal. Revista Brasileira de Engenharia Agrícola e Ambiental, v. 15, p. 277-288, 2011.

STANHILL, G.; COHEN, S. Global dimming: a review of the evidence for a widespread and significant reduction in global radiation with discussion of its probable causes and possible agricultural consequences. Agricultural and Forest Meteorology, v. 107, p. 255-78, 2001.

TERAMOTO, E.T; ESCOBEDO, J.F. Analysis of the annual frequency of the sky conditions in Botucatu, São Paulo, Brazil. Revista Brasileira de Engenharia Agrícola e Ambiental, v. 16, n. 9, p. 985-992, 2012.

TERAMOTO, E.T.; ESCOBEDO, J.F. Evolução diurna e anual da frequência das coberturas de céu em Botucatu/SP. Energia na agricultura, v. 27, n. 1, p. 37-52, 2012.

TIBA, C.; FRAIDEENRAICH, N.; GALLEGOS, H.G.; LYRA, F.J.M. Solar energy resource assessment - Brazil. Renewable Energy, v. 27, p. 383-400, 2002.

VARELLA, F.K.O.M.; CAVALIERO, C.K.N.; SILVA, E.P. A survey of the current photovoltaic equipment industry in Brazil. Renewable Energy, v. 34, p. 1801-1805, 2009.

WENXIAN, L.; WENFENG, G.; SHAOXUAN, P.; ENRONG, L. Ratios of global radiation on a tilted to horizontal surface for Yunnan Province, China. Energy, v. 20, n. 8, p. 723-728, 1995.

WONG, L.T.; CHOW, W.K. Solar radiation model. Applied Energy, v. 69, p. 191-224, 2001.

All the contents of this journal, except where otherwise noted, is licensed under a Creative Commons Attribution License CC-BY. 